\title{
EVALUACIÓN MORFOLÓGICA Y RENDIMIENTO DE LA VARIEDAD DE SOYA \\ (GLYCINE MAX L. MERRILL) INIAP 307 EN RESPUESTA A LA FERTILIZACIÓN ORGÁNICA Y MINERAL \\ MORPHOLOGICAL EVALUATION AND YIELD OF SOY (GLYCINE MAX L. MERRILL) INIAP 307 IN RESPONSE TO ORGANIC AND MINERAL FERTILIZATION
}

\author{
Christian Duran Mera, Mgtr. \\ https://orcid.org/0000-0003-2376-7522 \\ Universidad de Guayaquil, Guayaquil, Ecuador. \\ christian.duranm@ug.edu.ec \\ Manuel Carrillo Zenteno, Ph.D. \\ https://orcid.org/0000-0001-7062-8248 \\ Instituto Nacional de Investigaciones Agropecuarias, Quito, Ecuador. \\ manuel.carrillo@iniap.gob.ec \\ Jonathan López Bosquez, Mgtr. \\ https://orcid.org/0000-0002-6146-9748 \\ Universidad Técnica de Cotopaxi, Latacunga, Ecuador. \\ jonathan.lopez9292@utc.edu.ec \\ Miguel Balseca Castañeda, Ing. \\ https://orcid.org/0000-0002-1049-7407 \\ Universidad Técnica de Manabí, Portoviejo, Ecuador. \\ miguelbalck87@hotmail.com
}

\section{ARTÍCULO DE INVESTIGACIÓN}

Recibido: 25 de octubre de 2021

Aceptado: 29 de noviembre de 2021

\section{RESUMEN}

La soya es un cultivo de gran importancia económica por su alto aporte en proteína para la alimentación animal y humana. Los rendimientos de la soya dependen de las características genéticas y de que se satisfagan sus requerimientos nutricionales a través de la fertilización adecuada. Con la fertilización orgánica y mineral, se consigue ajustar recomendaciones nutricionales de acuerdo a las necesidades del cultivo, induciendo a un incremento del rendimiento el objetivo de la investigación fue evaluar el efecto de la fertilización orgánica y mineral sobre el comportamiento morfológico y rendimiento de la variedad de soya INIAP 307.

El trabajo constó de cinco tratamientos y tres repeticiones que se distribuyeron en un diseño de bloques completamente al azar, los resultados fueron sometidos a un análisis de varianza para determinar las diferencias estadísticas entre las medias de cada tratamiento utilizando la prueba de Tukey al $95 \%$ de probabilidad, las variables evaluadas fueron: altura de planta, días a floración, días a la maduración, altura de carga, acame, número de vainas por plantas, 
número de semillas por plantas, días a cosecha y rendimiento. Los resultados obtenidos mostraron que el mayor rendimiento fue con la aplicación de N-P-K-S con un promedio de 2296,67 kg ha-1. No se observó significancia en las características morfológicas y rendimiento con la aplicación de abono orgánico.

Palabras claves: agronomía, fertilizantes, producción, variedad

\section{ABSTRACT}

Soy is a crop of great economic importance due to its high protein content for animal and human nutrition. Soybean yields depend on genetic characteristics and that its nutritional requirements are met through proper fertilization. With organic and mineral fertilization, it is possible to adjust nutritional recommendations according to the needs of the crop, inducing an increase in yield. The objective of the research was to evaluate the effect of organic and mineral fertilization on the morphological behavior and yield of the variety. soybean INIAP 307. The work consisted of five treatments and three repetitions that were distributed in a completely randomized block design, the results were subjected to an analysis of variance to determine the statistical differences between the means of each treatment using the test of Tukey at 95\% probability, the variables evaluated were: plant height, days to flowering, days to maturation, loading height, lodging, number of pods per plant, number of seeds per plant, days to harvest and yield. The results obtained showed that the highest yield was with the application of N-P-K-S with an average of $2296.67 \mathrm{~kg}$ ha-1. No significance was observed in the morphological characteristics and performance with the application of organic fertilizer, so organic matter must be applied beforehand to consider, observe, determine the benefits on the physical, chemical, biological characteristics of the soil and so on. there is a better use of organic fertilizers and that at the time of sowing the plants have better growth and performance.

Keywords: agronomy, fertilizers, production, variety.

\section{INTRODUCCIÓN}

La soya (Glycine max L. Merrill) es una leguminosa de origen asiático y la principal oleaginosa a nivel mundial, debido a su alto porcentaje de proteína (35-50 \%) y aceite (15-25 $\%)$, por lo que constituye una fuente de proteína barata y de gran calidad, tanto para la alimentación del ganado como para la humana, sea como grano o en procesados como carne de soya, aceites, etc.; algunas industrias emplean para elaborar dulces, confitería, repostería y galletas; utilizando tanto el grano como la planta (Romero et al., 2013; Vergara et al., 2016). 
Los tres países más productores de soya a nivel mundial son: Brasil con una producción de $114,269,392$ t ha-1 en una superficie cosechada de $35,881,447$ ha, por lo que su rendimiento promedio fue de 3,2 t ha-1, Estados Unidos de América 96,793,180 t ha-1 en una superficie de 30,352,150 ha con un rendimiento de 3,2 t ha-1 y Argentina 55,263,891 t ha-1 con una superficie de 16,575,887 ha, rendimiento promedio de $3,3 \mathrm{t}$ ha-1. (FAOSTAT, 2019).

La producción de soya en el Ecuador obtuvo un rendimiento nacional promedio de 1,91 t ha-1 en una superficie de 17,056 ha. Este resultado fue obtenido con las variedades, INIAP 307, P34 y P32. La provincia del Guayas alcanzó la mayor producción, con rendimiento de 1,93 t ha-1 en una superficie de 12.608 ha; mientras que, en la provincia de Los Ríos registró una producción de 1,90 t ha-1 en una superficie de 4,421 ha (Ministerio de Agricultura y Ganadería, 2019).

Durante la época seca del año 2015, los productores de soya en el Ecuador utilizaron fertilizantes edáficos, aplicando en promedio $31 \mathrm{~kg}$ ha-1 de nitrógeno $(\mathrm{N}), 26 \mathrm{~kg}$ ha-1 de fósforo (P2O5) y $31 \mathrm{~kg}$ ha-1 de potasio (K2O). Estas cantidades fueron aplicadas a través de una serie de mezclas y fertilizantes compuestos y para el caso del $\mathrm{N}$ mayormente se usó Urea, es decir que el nivel de nutrición aplicado no satisface las necesidades del cultivo (Aguirre y Sarauz, 2015) a comparación de las recomendaciones dadas por el (INIAP, 2014) Nitrógeno (N) 46 kg ha-1, fósforo (P2O5) 34 kg ha-1 y potasio (K2O) 50 kg ha-1.

Para incrementar la productividad y mantener la estabilidad de los rendimientos de cosecha en el cultivo de soya, es fundamental asegurar la rentabilidad y contribuir al logro de sistemas sustentables por un mejor uso de los recursos, definiendo tácticas de manejo de una fertilización eficiente y rentable, que pueda afectar positivamente en el resultado económico a los productores soyeros (Nuñez et al., 2015).

Una alternativa viable y segura que permite obtener producciones sustentables de los cultivos constituye el empleo de abonos orgánicos; estos fertilizantes, garantizan la disminución o eliminación de los fertilizantes químicos, recuperan la fertilidad del suelo, incrementan la flora microbiana, la cual realiza una importante labor al descomponer los residuos orgánicos, convirtiéndolas en minerales, que pueden ser asimilados por las plantas durante su ciclo productivo (Travieso et al., 2018).

Debido al déficit de producción en el sector soyero, siendo un cultivo importante para la economía del país, se consideró la necesidad de determinar los factores que están influyendo en su producción, especialmente en la fertilización química y orgánica, según las necesidades nutricionales del cultivo, para que los productores no tengan bajos rendimientos de cosecha. 
Por esta razón el objetivo de la investigación fue evaluar el efecto de la fertilización orgánica y mineral sobre el comportamiento agronómico y rendimiento del cultivo de soya (Glycine max L. Merrill) variedad 307.

\section{MATERIALES Y MÉTODOS}

La presente investigación se la realizó en época seca durante los meses de junio - octubre del 2019 en la Estación Experimental Tropical Pichilingue del INIAP, ubicada en la provincia de Los Ríos perteneciente al cantón Mocache en el km 5 vía Quevedo - El Empalme. La Estación se encuentra localizada a una altitud promedio de $75 \mathrm{msnm}$ y posesionada geográficamente en las coordenadas $01^{\circ} 05^{\prime} 24^{\prime \prime}$ latitud Sur y $79^{\circ} 28^{\prime} 06^{\prime \prime}$ longitud Occidental. La temperatura media anual es de $26^{\circ} \mathrm{C}, 2.224 \mathrm{~mm}$ de precipitación, $86 \%$ de humedad relativa, 898 horas luz y la clase textural de suelo fue franco y su nivel de fertilidad se detalla en la tabla 1.

Tabla 1

Análisis de suelo del lote experimental, ubicado en la EETP-INIAP

\begin{tabular}{|c|c|c|c|c|c|c|c|c|c|c|c|c|}
\hline & Datos del lote & $\mathrm{NH}_{4}$ & $\mathbf{P}$ & K & $\mathrm{Ca}$ & Mg & $\mathbf{S}$ & $\mathbf{Z n}$ & $\mathrm{Cu}$ & $\mathrm{Fe}$ & Mn & B \\
\hline \multirow{3}{*}{ Sitio } & \multirow{3}{*}{ Nuevo lote Pichilingue } & \multicolumn{2}{|c|}{ ppm } & \multicolumn{3}{|c|}{$\mathrm{meq} / 100 \mathrm{ml}$} & \multicolumn{6}{|c|}{ ppm } \\
\hline & & \multirow{2}{*}{$13 \mathrm{~B}$} & 47 & 0,82 & 8 & 1,2 & 8 & 5,8 & 10,2 & 135 & \multirow{2}{*}{$17,1 \mathrm{~A}$} & \multirow{2}{*}{$1,02 \mathrm{~A}$} \\
\hline & & & A & A & M & $M$ & B & M & A & A & & \\
\hline M.O & $\mathrm{pH}$ & $\mathrm{Ca} / \mathrm{Mg}$ & \multicolumn{2}{|c|}{$\mathrm{Mg} / \mathrm{K}$} & \multicolumn{2}{|c|}{$\mathrm{Ca}+\mathrm{Mg} / \mathrm{K}$} & \multicolumn{2}{|c|}{$\Sigma$ bases } & \multicolumn{3}{|c|}{ Textura (\%) } & Clase \\
\hline$\%$ & MeAc RC & & & $1 \mathbf{1 0 0 n}$ & & & me & $00 \mathrm{ml}$ & arena & limo & arcilla & \\
\hline $3,6 \mathrm{M}$ & 5,6 & 6,6 & & & & & & & 35 & 46 & 19 & Franco \\
\hline
\end{tabular}

Nota: $\mathrm{MeAc}=$ medianamente ácido; $\mathrm{A}=\mathrm{Alto}, \mathrm{M}=$ medio, $\mathrm{B}=$ bajo. Fuente elaboración propia.

Se utilizó la semilla de soya variedad IINAIP 307 cuyas características agronómicas son: floración de 43 - 48 días; flor de color lila; 105 a 120 días a la cosecha; de 60 a 78 cm altura de planta; de 14 a $18 \mathrm{~cm}$ de altura de carga; resistente al volcamiento; con 3 a 8 ramas/planta; hojas de color verde oscuro; la forma del trifolio es oval; pubescencia de color café cobrizo; de 40 a 80 vainas/planta; $55-65 \%$ de vainas con tres semillas; con 64 a 145 semillas/planta; el color de grano es amarillento; con peso de 100 semillas de 16 a $20 \mathrm{~g}$, tolerante a insectos defoliadores, Cercospora, Peronospora, virosis; moderadamente resistente a Meloidogyne y con Rendimiento de $4467 \mathrm{~kg}$ ha-1 (INIAP, 2003).

El trabajo constó de cinco tratamientos con tres repeticiones, dando un total de 15 unidades experimentales que se distribuyeron en un diseño de bloques completamente al azar (DBCA). Las variables evaluadas fueron sometidas al análisis de varianza y para determinar las diferencias estadísticas entre las medias de cada tratamiento, se utilizó la prueba de Tukey al $95 \%$ de probabilidad y se utilizó el software estadístico infoStat 2019e. 
Tabla 2

Tratamientos evaluados en la investigación. Fertilización orgánica y mineral en la variedad de soya Iniap 307.

\begin{tabular}{cccccccc}
\hline \multirow{2}{*}{ Variedad } & \multirow{2}{*}{ Tratamientos } & $\mathbf{A O}$ & $\mathbf{N}$ & $\mathbf{P}_{\mathbf{2}} \mathbf{O}_{5}$ & $\mathbf{K}_{\mathbf{2}} \mathbf{O}$ & $\mathbf{S}$ & $\mathbf{M g O}$ \\
\cline { 3 - 8 } & & 0 & 0 & 0 & 0 & 0 & 0 \\
\hline \multirow{3}{*}{ Iniap 307 } & Testigo & 2000 & 0 & 0 & 0 & 0 & 0 \\
& Abono Orgánico & 0 & 46 & 23 & 50 & 0 & 0 \\
& N-P-K & 0 & 46 & 23 & 50 & 24 & 0 \\
& N-P-K-S & 0 & 46 & 23 & 50 & 24 & 28 \\
\hline
\end{tabular}

Fuente: Elaboración propia

La preparación del terreno se la realizó con la utilización de dos pases de rastra, y posteriormente, se realizó el estaquillado del área para la identificación de las parcelas con cada uno de sus tratamientos y repeticiones.

Antes de realizar la siembra se trató la semilla con Tiametosan y Carboxim + Thiram, además se realizó la inoculación de la semilla con Bradyrhizobium japonicum, luego se procedió a realizar la siembra de forma manual con un distanciamiento de $0,45 \mathrm{~m}$ entre hilera distribuyendo 18 semillas por metro lineal dando un total de 400.000 plantas/ha.

El control de malezas se lo realizó manualmente con la utilización de machete, para el control de insectos plagas se aplicó Spinetoram (100cc ha-1) a los 27 días después de la siembra (DDS), Acetamiprid (200g ha-1) a los 41 DDS; para el control de enfermedades, se aplicó Picoxystrobin (250cc ha-1) a los a los 27 DDS y Azoxistrobina + Difenoconazole (300cc ha-1) a los 45 DDS.

La fertilización se la realizó de acuerdo a las dosis de fertilizantes según el requerimiento nutricional del cultivo las cuales fueron: $46 \mathrm{~kg}$ ha-1 N; $23 \mathrm{~kg}$ ha-1 P2O5; $50 \mathrm{~kg}$ ha-1 K2O; 24 $\mathrm{kg}$ ha-1 S y $28 \mathrm{~kg}$ ha-1 MgO, como fuentes de fertilizantes se usaron productos disponibles en el mercado y de fácil acceso al productor como se menciona en la tabla 3. 
Tabla 3

Fuentes, elementos y composición del abono orgánico y mineral empleados en la fertilización de la investigación.

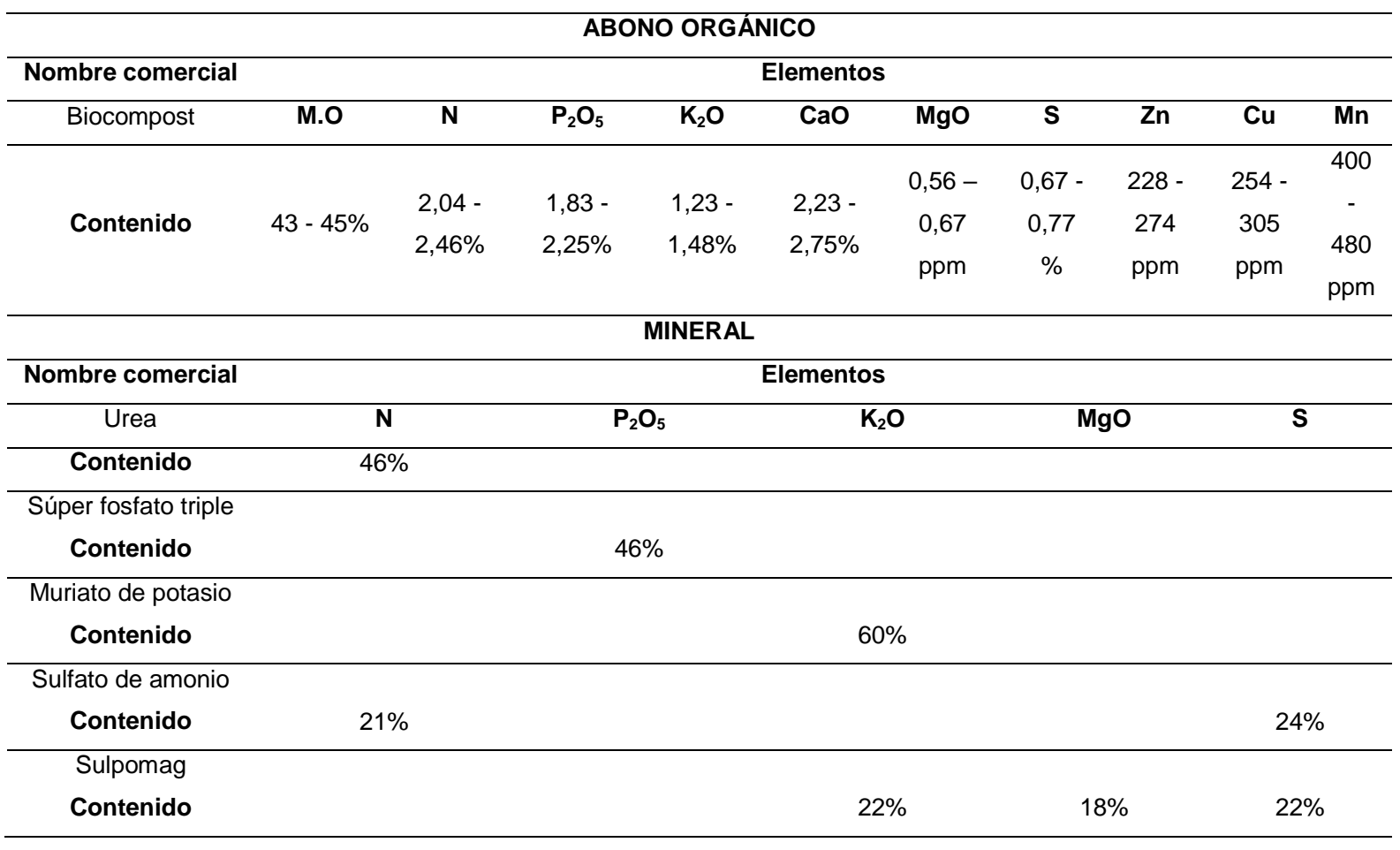

Fuente: Elaboración propia

Las aplicaciones fueron manuales en el caso de las fuentes minerales se realizó una mezcla uniforme, colocando el abono orgánico (100\% antes de la siembra e incorporado con pase de rastra), fraccionando el $\mathrm{N}$ en dos aplicaciones ( $25 \%$ y $75 \%$ a los 15 y 25 DDS), $P$ (100\% a los 7 DDS), K, S y Mg (100\% a los 15 DDS).

\section{Variables evaluadas}

\section{Altura de planta (cm)}

Esta variable se la determinó a la cosecha en 10 plantas tomadas al azar, midiendo con la ayuda de una cinta métrica desde el nivel del suelo, hasta la yema apical de cada planta.

\section{Días a la floración}

Se registró desde la fecha de siembra hasta cuando las plantas presentaron mínimo el 50 $\%$ de flores abiertas.

\section{Días a la maduración}

Esta variable se la registró de acuerdo al número de días transcurridos desde la fecha de siembra hasta cuando el $95 \%$ de sus vainas ubicadas en el tallo principal llegan a tener un color marrón gris y las plantas tornan un color amarillento. 


\section{Altura de carga (cm)}

El registro de esta variable se realizó en 10 plantas, midiendo desde el cuello de la planta hasta el punto de la inserción de la primera vaina.

\section{Acame}

Se evaluó en cada uno de los tratamientos en el momento previo a la cosecha utilizando la escala de 1 a 5, propuesta por el Instituto Internacional de la Soya (INTSOY, 1984). Escala 1-5:

1.- Plantas erectas.

2.- Plantas ligeramente inclinadas o poco tendidas (10\%).

3.- Plantas moderadamente inclinadas (25 a $50 \%$ ).

4.- Plantas considerablemente inclinadas (51 a $80 \%$ ).

5.- Todas las plantas totalmente tendidas

\section{Número de vaina por planta}

Se tomaron 10 plantas al azar y luego se procedió a contar el número total de vainas existentes en cada una de las plantas.

\section{Número de semillas por plantas}

En esta variable se tomaron 10 plantas al azar y luego se contó el total de semillas por vaina.

\section{Días a la cosecha}

Esta variable se registró días antes de la cosecha, cuando las plantas y vainas presentaron los síntomas característicos de la madurez fisiológica (defoliación de hojas y secamiento total de vainas).

\section{Rendimiento $\mathrm{kg}$ ha-1}

El rendimiento se obtuvo de acuerdo a las semillas cosechadas en el área útil de cada parcela experimental, para lo cual se transformó a $\mathrm{kg}$ ha-1, ajustado al $13 \%$ de humedad. Se aplicó la siguiente fórmula:

$$
\text { P Aj }\left(\frac{\mathrm{kg}}{\mathrm{ha}}\right)=\frac{\operatorname{Pac} \times(100-\mathrm{Hac})}{100-\mathrm{Hd}} \times \frac{10.000 \mathrm{~m} 2}{\text { Área útil }}
$$

Dónde: $P$ Aj = peso ajustado.

$\mathrm{Pac}=$ peso actual.

$\mathrm{Hac}=$ humedad actual.

$\mathrm{Hd}=$ humedad deseada. 


\section{RESULTADOS}

De acuerdo a las evaluaciones realizadas del comportamiento morfológico de la soya INIAP 307 y la determinación del rendimiento se obtuvieron los siguientes resultados.

No se observaron diferencias significativas para ninguna de las variables de altura de plantas $(\mathrm{cm})$, días a la floración, días a la maduración y altura de carga $(\mathrm{cm})$ por efecto de la fertilización orgánica y mineral como se refleja en la tabla 3.

Tabla 3

Altura de planta (cm), días a la floración, días a la maduración, altura de carga $(\mathrm{cm})$, por efecto de la nutrición orgánica y mineral del cultivo de soya (Glycine max L. Merrill) variedad 307. Mocache, época seca 2019.

\begin{tabular}{ccccc}
\hline Tratamientos & Altura de planta $\mathbf{( c m})$ & $\begin{array}{c}\text { Días a la } \\
\text { floración }\end{array}$ & $\begin{array}{c}\text { Días a la } \\
\text { maduración }\end{array}$ & $\begin{array}{c}\text { Altura de } \\
\text { carga }(\mathbf{c m})\end{array}$ \\
\hline T & $72,00 \mathrm{a}$ & $39,67 \mathrm{a}$ & $92,00 \mathrm{a}$ & $11,33 \mathrm{a}$ \\
A.O & $71,67 \mathrm{a}$ & $40,67 \mathrm{a}$ & $94,33 \mathrm{a}$ & $8,33 \mathrm{a}$ \\
N-P-K & $71,33 \mathrm{a}$ & $38,33 \mathrm{a}$ & $93,67 \mathrm{a}$ & $8,67 \mathrm{a}$ \\
N-P-K-S & $73,67 \mathrm{a}$ & $40,67 \mathrm{a}$ & $94,33 \mathrm{a}$ & $10,00 \mathrm{a}$ \\
N-P-K-S-Mg & $73,00 \mathrm{a}$ & $40,00 \mathrm{a}$ & $95,00 \mathrm{a}$ & $9,33 \mathrm{a}$ \\
\hline C.V (\%) & 6,12 & 2,34 & 1,51 & 17,76 \\
\hline p-valor & $0,9613 \mathrm{NS}$ & $0,0622 \mathrm{NS}$ & $0,1758 \mathrm{NS}$ & $0,2773 \mathrm{NS}$ \\
\hline
\end{tabular}

NS No significativo al $5 \%$ de probabilidad. Medias dentro de columnas con letras distintas son estadísticamente diferentes de acuerdo al test de Tukey al $5 \%$ de probabilidad. Fuente: Elaboración propia

En cuanto a las variables Acame, número de semillas por planta y días a la cosecha no presentaron diferencias significativas; sin embargo, para la variable número de vainas por planta presentó diferencia significativa con el tratamiento N-P-K logrando alcanzar un promedio de 46,33 vainas por plantas y el tratamiento testigo presentó el menor promedio con 35,67 vainas por plantas como se muestra en la tabla 4 . 
Tabla 4

Acame, número de vainas por planta, número de semillas por planta, días a la cosecha, por efecto de la nutrición orgánica y mineral del cultivo de soya (Glycine max L. Merrill) variedad 307. Mocache, época seca 2019.

\begin{tabular}{ccccc}
\hline Tratamientos & Acame & $\begin{array}{c}\text { Número de } \\
\text { vainas por } \\
\text { planta }\end{array}$ & $\begin{array}{c}\text { Número de } \\
\text { semillas por } \\
\text { planta }\end{array}$ & $\begin{array}{c}\text { Días a la } \\
\text { cosecha }\end{array}$ \\
\hline T & $2,33 \mathrm{a}$ & $35,67 \mathrm{a}$ & $67,33 \mathrm{a}$ & $110,33 \mathrm{a}$ \\
A.O & $2,67 \mathrm{a}$ & $41,67 \mathrm{ab}$ & $80,00 \mathrm{a}$ & $112,33 \mathrm{a}$ \\
N-P-K & $2,67 \mathrm{a}$ & $46,33 \mathrm{~b}$ & $72,33 \mathrm{a}$ & $112,00 \mathrm{a}$ \\
N-P-K-S & $3,00 \mathrm{a}$ & $43,00 \mathrm{~b}$ & $84,67 \mathrm{a}$ & $111,67 \mathrm{a}$ \\
N-P-K-S-Mg & $2,33 \mathrm{a}$ & $41,33 \mathrm{ab}$ & $68,00 \mathrm{a}$ & $111,00 \mathrm{a}$ \\
\hline C.V (\%) & 31,40 & 6,11 & 23,41 & 0,90 \\
\hline p-valor & $0,8383 \mathrm{NS}$ & 0,0062 ** & $0,6889 \mathrm{NS}$ & $0,1814 \mathrm{NS}$ \\
\hline
\end{tabular}

${ }^{\mathrm{NS}}$ No significativo al $5 \%$ de probabilidades del error ${ }^{* *}$ Altamente significativo al $5 \%$ de probabilidad. Medias dentro de columnas con letras distintas son estadísticamente diferentes de acuerdo al test de Tukey al $5 \%$ de probabilidad. Fuente: Elaboración propia.

Para la variable rendimiento $\mathrm{kg}$ ha-1 se puede observar estadísticamente diferencias significativas obteniéndose los mayores valores con los tratamientos N-P-K-S y N-P-K-S-Mg logrando superar un rendimiento de 2 t ha-1 como se refleja en la figura 1.

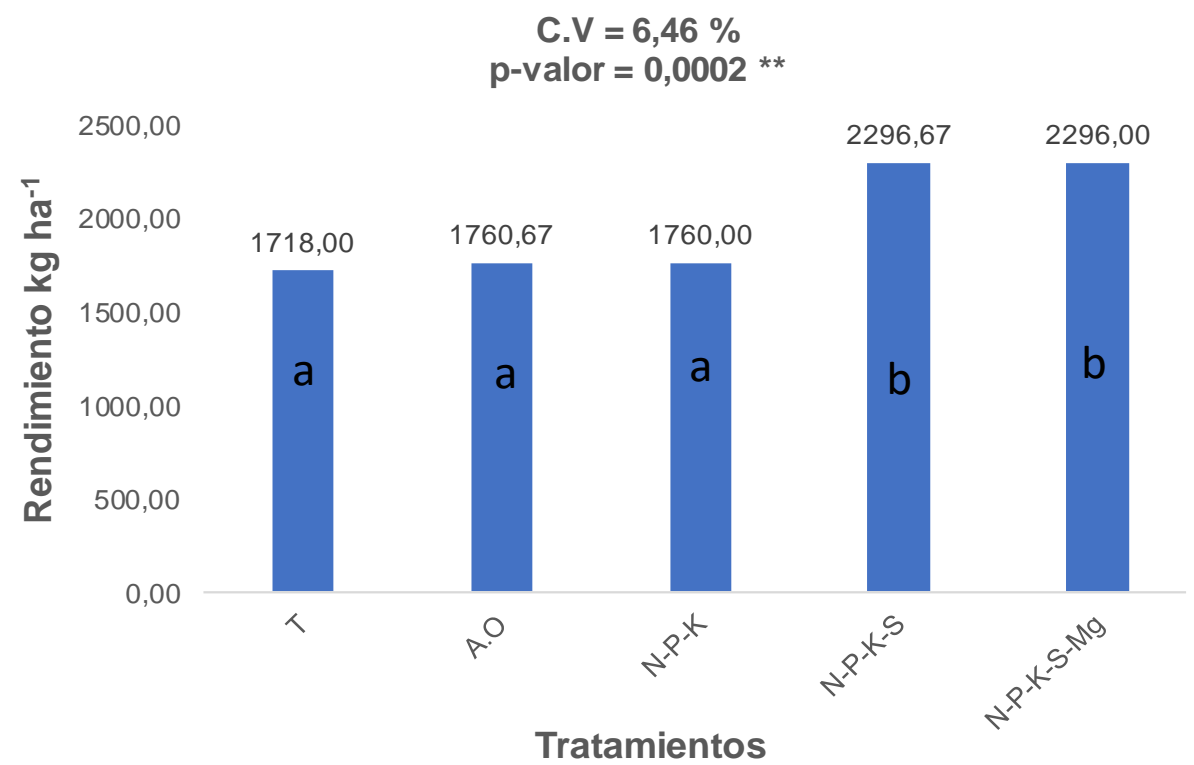

Figura 1. Rendimiento $\mathrm{kg} \mathrm{ha}^{-1}$ por efecto de la nutrición orgánica y mineral del cultivo de soya (Glycine $\max$ L. Merrill) variedad 307. Mocache, época seca 2019. Nota: Letras distintas son estadísticamente diferentes de acuerdo al test de Tukey al $5 \%$ de probabilidad. ${ }^{* *}$ Altamente significativo al $5 \%$ de probabilidad. Fuente: Elaboración propia. 


\section{DISCUSIÓN}

La fertilización orgánica y mineral no influyó en la altura de planta, días a la floración, días a la maduración, altura de carga, acame, número de semillas por planta, días a la cosecha, debido a que la variedad de soya INIAP 307 expresó su genética en cuanto a sus características morfológicas como lo indica Choez et al. (2020) que describe a la variedad INIAP 307, al igual con el trabajo investigativo de Campi (2019) evaluó densidades poblacionales, tipos de siembra y fertilización edáfica en época seca con la variedad de soya INIAP 307 en la finca "La María", localizada en el Km 7 de la vía Quevedo - El Empalme y aplicó como fuente de fertilizante 8-20-20, en dosis de $200 \mathrm{Kg} \mathrm{ha}-1$, obtuvo valores no tan superiores a los reportados en esta investigación: 75,47 (cm) para la altura de planta, días a la floración 46,00 y altura de carga 13,78 (cm).

Por otro lado, se concuerdan valores reportados por Manzaba (2015) usando $20 \mathrm{~kg}$ ha-1 Nitrofoska $+100 \mathrm{~kg}$ de Sustrato: $2,75 \%$ de acame, 109 días a la cosecha; al igual con la investigación de Cabanilla (2015) aplicó 10-30-10 en dosis de 50 kg ha-1, Abono Azul $50 \mathrm{~kg}$ ha-1, Urea $100 \mathrm{~kg}$ ha-1, Muriato de Potasio $50 \mathrm{~kg}$ ha-1 y un abono foliar Metalosato $1 \mathrm{I}$ ha-1, obtuvo un promedio de 68 semillas por plantas.

Para el número de vainas con la aplicación de N-P-K se logró alcanzar un promedio de 46,33 vainas por plantas, valor superior a la investigación de Freire (2018) que al utilizar un tratamiento de Riego + NPK alcanzó un promedio de 36,00 vainas/planta. Con el tratamiento de abono orgánico se obtuvo un promedio de 41,67, valor cercano a lo reportado por Travieso et al., (2018) donde evaluó diferentes dosis de abonos orgánicos alcanzando un promedio de 42,12 vainas/planta con la aplicación de estiércol vacuno 40 t ha-1.

El efecto de la fertilización mineral sobre el rendimiento fue superior con respecto a las plantas que recibieron el abono orgánico, siendo los tratamientos N-P-K-S y N-P-K-S-Mg superando un promedio de 2 t ha-1, este rendimiento concuerda con de Freire (2018) utilizando Riego + NPK, por otro lado se supera el valor reportado por Ayala (2011) utilizó 50 $\mathrm{kg}$ ha-1 urea; $50 \mathrm{~kg}$ ha-1 superfosfato triple; $100 \mathrm{~kg}$ ha-1 muriato de potasio y $100 \mathrm{~kg}$ ha-1 Nitrofoska; pero se discrepa con lo reportado por Vinueza (2016) en Taura - Guayas, obtuvo más de 4,5 tha-1 resultado que puede deberse a que es una zona de mayor luminosidad que contrastan con la zona de Mocache. Con abono orgánico se obtuvo 1,7 t ha-1, este promedio concuerda con Travieso et al., (2018) obtuvo 1,72 t ha-1 con la aplicación de 20 t ha-1 de estiércol vacuno, existe un valor cercano a los reportados por Medina y Blandón (2010) utilizan una aplicación con abonos orgánicos y obtuvieron un rendimiento de 1,8 t ha-1. 
Cruz et al., (2014) menciona que el uso de abonos orgánicos es un fertilizante natural de alta calidad y no posee efectos nocivos, su efecto en la mejora del suelo promueve el crecimiento y un mejor rendimiento en los cultivos, sin embargo Garro (2016) manifiesta que los abonos orgánicos son alternativas que permiten solventar deficiencias de fertilidad de cada suelo o bien en función de las necesidades del cultivo, todo esto sin dejar de considerar siempre la vida microbiana, la que es muy importante en el aprovechamiento óptimo de los abonos.

\section{CONCLUSIONES}

Se consiguió superar el rendimiento de 2 t ha- 1 de grano de soya al $13 \%$ de humedad, aplicando N-P-K-S y N-P-K-S-Mg, resultando los mejores tratamientos de fertilización.

No se observó significancia en las características morfológicas y rendimiento con la aplicación de abono orgánico, por lo que se debe aplicar materia orgánica con anterioridad para considerar, observar, determinar los beneficios sobre las características físicas, químicas, biológicas del suelo y así de esa manera exista un mejor aprovechamiento de los abonos orgánicos y que al momento de realizar una siembra las plantas tengan un mejor crecimiento y rendimiento.

\section{REFERENCIAS BIBLIOGRÁFICAS}

Aguirre, M; Sarauz, S. (2015). Rendimientos y características de soya en Ecuador verano 2015. https://docplayer.es/67773462-Rendimientos-y-caracteristicas-de-soya-en-elecuador-verano-2015-julio-octubre.html

Ayala, C. (2011). Evaluación de 12 cultivares de soya (Glycine max (L).), en comparación de testigos locales en la zona de Pueblo Nuevo, Provincia de Los Ríos. (Tesis de Pregrado, Universidad de Guayaquil). http://repositorio.ug.edu.ec/bitstream/redug/8161/1/tesis\%20carlos.pdf

Campi, R. (2019). Efecto de las densidades poblacionales, tipos de siembra y fertilización edáfica en el cultivo de soya (Glycine max L.) sembrado en la época seca, zona de Quevedo. (Tesis de Pregrado, Universidad Técnica Estatal de Quevedo). https://repositorio.uteq.edu.ec/bitstream/43000/3630/1/T-UTEQ-0166.pdf

Cabanilla, L. (2015). Evaluación agronómica de materiales de soya (Glycine max. (L) Merril) de hilium claro (Tesis de Pregrado, Universidad de Guayaquil) Guayaquil, Ecuador. http://repositorio.ug.edu.ec/bitstream/redug/8640/1/Cabanilla\%20Guaman\%20Leyddi \%20Janneth.pdf 
Cruz, E., Can, A., Bugarín, R., Pineda, J., Flores, R., Juárez, P. \& Alejo, G. (2014). Concentración nutrimental foliar y crecimiento de chile serrano en función de la solución nutritiva y el sustrato. Revista Fitotecnia Mexicana 37 (3), 289-295. http://www.scielo.org.mx/scielo.php?script=sci_arttext\&pid=S018773802014000300016

Choez-Quiroz, V. I., Paz, C. L., Valdés-Carmenate, R., \& Paredes-Pacheco, M. (2020). INIAP 307: Variedad de soya (Glycine max L. Merril) en Ecuador. Cultivos Tropicales, 41(3).

FAOSTAT. (2019). Cultivos y productos de ganadería Cantidades de producción de Soja por país. https://www.fao.org/faostat/es/\#data/QCL/visualize

Freire, J. (2018). Determinación del efecto del riego y la fertilización en el rendimiento del cultivo de soya (Glycine max) en la zona de Mocache, (Tesis de Pregrado, Universidad Técnica Estatal de Quevedo). https://repositorio.uteq.edu.ec/bitstream/43000/3310/1/T-UTEQ-0140.pdf

Garro, J., E. (2016). El suelo y los abonos orgánicos. Costa Rica. Instituto Nacional de Innovación y Transferencia en Tecnología Agropecuaria. C.R.: INTA, 106. http://www.mag.go.cr/bibliotecavirtual/F04-10872.pdf

INTSOY. (1984). Escala Internacional del INTSOY. Departamento de Agronomía, Universidad de Illinois, EE. UU. http://www.oas.org/dsd/environmentlaw/trade/soja/librosoja.pdf

INIAP. (2003). Iniap 307 nueva variedad de soya de gran rendimiento y resistente a acame. https://repositorio.iniap.gob.ec/bitstream/41000/1993/1/iniaplsbd313.pdf

INIAP. 2014.

Nutrición:

soya. http://tecnologia.iniap.gob.ec/images/rubros/contenido/soya/5nutricion.pdf

Ministerio de Agricultura y Ganadería. (2019). Informe de rendimientos objetivos soya 2019: $12 \%$ humedad, $1 \%$ impureza. https://fliphtml5.com/ijia/cczg/basic

Medina; Blandón, L. (2010). Efecto de fertilizantes orgánicos y sintéticos en el crecimiento y rendimiento del cultivo de soya (Glycine max L Merrill), El Plantel, Masaya, 2009 (Tesis de Pregrado, Universidad Nacional Agraria). https://repositorio.una.edu.ni/2118/

Nuñez, A; Lamothe, GA; Sawchik, J. (2015). Manejo de la fertilización en el cultivo de soja, INIA Treinta y Tres - Estación Experimental del Este Arroz-Soya, (4), 1. http://www.ainfo.inia.uy/digital/bitstream/item/7922/1/ad-748-cap-6-p.21-24.pdf 
Manzaba, S. (2015). Rendimiento y características agronómicas de ocho variedades de soya (Glycine max L.) en la zona central del litoral ecuatoriano, (Tesis de Pregrado, Universidad Técnica de Qstatal Quevedo). https://repositorio.uteq.edu.ec/bitstream/43000/2398/1/T-UTEQ-0308.pdf

Romero, A; Ruz, R; González, M. (2013). Evaluación de siete cultivares de soya (Glycine $\max$ ) en las condiciones edafoclimáticas del municipio Majibacoa, Pastos y Forrajes, Vol. 36, No. 5 p. http://scielo.sld.cu/scielo.php?script=sci_arttext\&pid=S086403942013000400006

Travieso, MG; García, TL; Pupo Blanco, YG; Tamayo López, LA; Gómez Machado, R; Galindo Jaguaco, WR; Lescay Batista, E. (2018). Respuesta productiva de (Glycine max) a diferentes dosis de abonos orgánicos en suelo Pardo Sialítico. Centro Agrícola 45(3):37-43. http://scielo.sld.cu/scielo.php?script=sci_arttext\&pid=S025357852018000300037

Vergara, NS; Orellana, FR; Vizueta, VH; Mata, DA; Bernal, DA; San Andres, PR. (2016). E cultivo de soya y su importancia para el Ecuador. INNOVA Research Journal 1(12):7785. https://revistas.uide.edu.ec/index.php/innova/article/view/110/143

Vinueza. (2016). Evaluación comparativa de materiales de soya tipo aceitera, evaluadas en la zona de Taura, Provincia del Guayas. (Tesis de Pregrado, Universidad Católica de Santiago de Guayaquil). http://repositorio.ucsg.edu.ec/bitstream/3317/7022/1/TUCSG-PRE-TEC-AGRO-108.pdf 